\title{
Multifractality and Multiscaling in two dimensional fragmentation
}

\author{
M K Hassan ${ }^{\mathrm{ab}}$ and G J Rodgers ${ }^{\mathrm{a}}$ \\ a Department of Physics, Brunel University, Uxbridge, Middlesex UB8 3PH, UK \\ b Department of Physics, Shahjalal Science and Technology University, Sylhet, Bangladesh
}

\begin{abstract}
We consider two models (A and B) which can describe both two dimensional fragmentation and stochastic fractals. Model A exhibits multifractality on a unique support when describing a fragmentation process and on one of infinitely many possible supports when describing stochastic fractals. Model B obeys simple scaling.
\end{abstract}

PACS numbers: 05.20-y,02.50-r

Keywords: Fragmentation, stochastic, fractal, multifractal

E-mail: Kamrul.Hassan@brunel.ac.uk and G.J.Rodgers@brunel.ac.uk 
Fragmentation is an irreversible kinetic process in which a collection of fragments are sequentially broken. There have been a number of different analytical approaches to the problem of the kinetics of fragmentation of one dimensional particles. These have included using the maximum entropy method [1], using statistical and combinatorial arguments $[2,3]$ and using a kinetic equation. It is the kinetic equation approach, developed by Filippov [4] after it's original proposal by Kolmogorov [5], that has provided most of our theoretical understanding. This has resulted in numerous exact and explicit solutions for the particle size distribution function [6,7], in addition to scaling solutions $[8,9]$.

In one dimension the size or mass of the particles is the only dynamical quantity of interest. However, there has been a recent theoretical interest [10-12,20] in the kinetics of fragmentation of multidimensional objects. This is motivated by a desire to move away from characterising particles solely by their volume or equivalently, mass and towards an understanding of the physical role played by shape in the fragmentation of particles. In reality, particles are identified by their shape.

The studies of fragmentation phenomenon in two dimensions [10-12, 20] have revealed unexpectedly rich patterns with interesting and novel statistics. We attempt to clarify the origin of this behaviour and invoke the idea of multifractality to characterise such pattern. We find that some fragmentation rules do not have a single measure support instead they have an infinite number. Each measure yields different spectrum of exponents to characterize the system. We also consider a second model which exhibits simple scaling and we seek to explain the difference between these models.

The obvious extension of the rate equation to two dimensions [10-13]] is

$$
\begin{aligned}
\frac{\partial f(x, y ; t)}{\partial t}= & -f(x, y ; t) \int_{0}^{x} d x_{1} \int_{0}^{y} d y_{1} F\left(x_{1}, y_{1}, x, y\right)+ \\
& s \int_{x}^{\infty} d x_{1} \int_{y}^{\infty} d y_{1} f\left(x_{1}, y_{1} ; t\right) F\left(x, y, x_{1}, y_{1}\right)
\end{aligned}
$$

where $f(x, y ; t)$ is the concentration of particles of sides $x$ and $y$ at time $t$ and $s=1,2,3$ or 4. $F\left(x_{1}, y_{1}, x, y\right)$ describes the rate with which objects having sides $x$ and $y$ break to produce fragments of sides $x_{1}, x-x_{1}$ and $y_{1}, y-y_{1}$.

The two integrals over the two variables implies that the two orthogonal cracks are placed on an objects such that the cracks are equal and parallel to the sides. Evidently they will produce four new fragments at each time step to describe fragmentation process $(s=4)$. However, we can choose $s=1,2,3$ which simply implies that $s$ fragments are kept and $(4-s)$ fragments are removed at each time step. This process creates a stochastic fractals [14,15]] at long times. In this letter, we consider our two variables $x$ and $y$ to be lengths. This is in contrast to [12], where one variable was associated with energy and the other with mass.

We choose to study a homogeneous rate kernel [12],

$$
F\left(x_{1}, y_{1}, x, y\right)=x^{\beta_{1}} y^{\beta_{2}}
$$

This model (called A in the abstract) describes a system in which particles are selected for fragmentation with a rate determined by their area and shape. The relative importance of area and shape is determined by $\beta_{1}$ and $\beta_{2}$. Once a particles has been chosen for fragmentation, products of any area and shape are equally likely so that the daughter distribution is Poisson. Substituting this choice of kernel into the rate equation yields 


$$
\frac{\partial f(x, y, ; t)}{\partial t}=-x^{\beta_{1}+1} y^{\beta_{2}+1} f(x, y ; t)+s \int_{x}^{\infty} \int_{y}^{\infty} d x_{1} d y_{1} x_{1}^{\beta_{1}} y_{1}^{\beta_{2}} f\left(x_{1}, y_{1} ; t\right)
$$

We now define the moments of the probability distribution function $f(x, y ; t)$ as

$$
M_{m, n}(t)=\int_{0}^{\infty} \int_{0}^{\infty} d x d y x^{m-1} y^{n-1} f(x, y ; t)
$$

We can then obtain a rate equation for the moments,

$$
\frac{\partial M_{m, n}(t)}{\partial t}=\left(\frac{s}{m n}-1\right) M_{m+\beta_{1}+1, n+\beta_{2}+1}(t)
$$

An interesting feature of the above equation is that there are infinitely many conserved (time independent) moments. Moments $M_{m, n}(t)$ that are conserved satisfy $m n=s$. This simply reflects the fact that fragments with a given area can have an infinite number of different shapes.

Using Charlesby's method, the moment equation can be iterated to get all the derivatives of the moments $[11,12]$. These can then be substituted into a Taylor series expansion of $M_{m, n}(t)$ about $t=0$ to give

$$
M_{m, n}(t)={ }_{2} F_{2}\left(a_{1}, a_{2} ; b_{1}, b_{2} ;-t\right)
$$

${ }_{2} F_{2}$ is a generalised hypergeometric function [15], where

$$
a_{1 / 2}=\frac{m}{2\left(\beta_{1}+1\right)}+\frac{n}{2\left(\beta_{2}+1\right)} \mp \sqrt{\frac{\left(m\left(\beta_{2}+1\right)-n\left(\beta_{1}+1\right)\right)^{2}+4 s\left(\beta_{1}+1\right)\left(\beta_{2}+1\right)}{4\left(\beta_{1}+1\right)^{2}\left(\beta_{2}+1\right)^{2}}}
$$

and

$$
\begin{aligned}
& b_{1}=\frac{m}{\left(\beta_{1}+1\right)} \\
& b_{2}=\frac{n}{\left(\beta_{2}+1\right)}
\end{aligned}
$$

We are only interested in the long time behaviour of the moments. The asymptotic expansion of the generalised hypergeometric function for large time $t$ gives

$$
M_{m, n}(t) \approx \frac{\Gamma\left(b_{1}\right) \Gamma\left(b_{2}\right) \Gamma\left(a_{1}-a_{2}\right)}{\Gamma\left(a_{2}\right) \Gamma\left(b_{1}-a_{1}\right) \Gamma\left(b_{2}-a_{1}\right)} t^{-a_{1}}
$$

For $s=4$ the conserved moments can be written as $M_{m^{*}, \frac{4}{m^{*}}}(t)$ where, $m^{*}$ is any number. We will only consider $m^{*}$ positive. Obviously, $M_{2,2}(t)$ can be identifyed as the area of the system. For convenience, we choose $\beta_{1}=\beta_{2}=\beta$ since it does not change the physics of the mechanism under investigation.

We may choose to associate each hidden conserved quantity of the $s=4$ process with the set of points in $\mathcal{R}^{2}$. This space can then be subdivided into boxes of size

$$
\delta_{m *}=\sqrt{\left.\frac{M_{m^{*}, \frac{4}{m^{*}}}(t)}{M_{1,1}(t)}\right)} .
$$


such that $\mu_{i}\left(\delta_{m^{*}}\right)$ denotes the measure within the $i^{t h}$ box that depends on the choice of $s$. This choice of $\delta_{m^{*}}$ ensures that for a pure fragmentation process $(s=4)$, we recover the complete set of points in $\mathcal{R}^{2}$. We now express the moment $M_{m, 1}(t)$ in terms of $\delta_{m^{*}}$

$$
M_{m, 1}\left(\delta_{m^{*}}\right) \sim \delta_{m^{*}}^{-\gamma\left(m^{*}\right)\left(\sqrt{(m-1)^{2}+4 s}-m-1\right)}
$$

where

$$
\gamma\left(m^{*}\right)=\frac{2}{\left(m^{*}+\frac{4}{m^{*}}\right)-\sqrt{\left(m^{*}-\frac{4}{m^{*}}\right)^{2}+4 s}-2+\sqrt{4 s}}
$$

When $m=1$, the exponent of equation (13) gives the Hausdorff-Besicovitch dimension $D_{f}$. Note that $\gamma\left(m^{*}\right)=\gamma\left(4 / m^{*}\right)$ and that in the limit $m^{*} \rightarrow \infty$, when $s>1, \gamma\left(m^{*}\right) \rightarrow 1 /(\sqrt{s}-1)$. We also see that for $s=4, \gamma\left(m^{*}\right)=1$ and $D_{f}=2$, independent of $m^{*}$, as we ensured with our choice of $\delta_{m^{*}}$. However, for $1<s<4$ there exist infinitely many supports for different values of $m^{*}$ and each is subdevided by a corresponding $\delta_{m^{*}}$. We also can write the $d$-measure of the weighted box number $M_{m, 1}(t)$ as

$$
M_{m, 1}\left(d, \delta_{m^{*}}\right)=\sum_{i} \mu_{i}^{k(m)} \delta_{m^{*}}^{d}=N\left(k(m), \delta_{m^{*}}\right) \delta_{m *}^{d}
$$

Where $N\left(k(m), \delta_{m^{*}}^{d}\right)$ is the $k(m)^{t h}$ moment of the measure such that $N\left(0, \delta_{m^{*}}\right)$ is the number of boxes require to cover the support of dimension $d=D_{f}$. Hence we can write the weighted box number as

$$
N\left(k(m), \delta_{m^{*}}^{d}\right)=\sum_{i} \mu_{i}^{k(m)} \sim \delta_{m^{*}}^{-\tau(k(m))}
$$

We thus see that $M_{m, 1}\left(\delta_{m^{*}}\right)$ can be partioned into boxes of sides $\delta_{m^{*}}$ such that the probabilities $\left\{\mu_{i}\right\}$ are normalised if we let

$$
m=1+(s-1) k
$$

when $\delta_{m^{*}} \rightarrow 0$ we require that the measure $M_{m, 1}\left(d, \delta_{m^{*}}\right)$ tends to a finite value. This occurs when $d=\tau(k)$. Combining (13),(15) and (17) gives the mass exponent $\tau(k)$ as

$$
\tau(k)=\gamma\left(m^{*}\right)\left\{\sqrt{(s-1)^{2} k^{2}+4 s}-(s-1) k-2\right\}
$$

This expression meets some essential requirement; namely $\tau(0)$ is the dimension of the support and $\tau(1)=0$.

We thus see that there exist a spectrum of mass exponents $\tau(k)$ that characterise the distribution of the particle size distribution. The mass exponent is nonlinear which indicates the existence of a fractal subset for each support whether or not the support itself is fractal. To find this fractal subset we use the usual Legendre transform of the independent variables $\tau$ and $k$ to the independent variable $\alpha$ and $f(\alpha(k))$;

$$
\alpha(k)=-\frac{d \tau(k)}{d k}
$$

and

$$
f(\alpha(k))=k \alpha(k)+\tau(k)
$$


These relations yield

$$
\alpha(k)=\gamma\left(m^{*}\right) \frac{(s-1) \sqrt{(s-1)^{2} k^{2}+4 s}-(s-1)^{2} k}{\sqrt{(s-1)^{2} k^{2}+4 s}}
$$

and

$$
f(\alpha(k))=\gamma\left(m^{*}\right) \frac{4 s-2 \sqrt{(s-1)^{2} k^{2}+4 s}}{\sqrt{\left(s-1^{2} k^{2}+4 s\right.}}
$$

It is interesting to note that all the quantities of interest, $\gamma\left(m^{*}\right), \tau(k)$ and $f(\alpha(k))$ are independent of $\beta$ when $\beta_{1}=\beta_{2}=\beta$. When $\beta_{1} \neq \beta_{2}$ all the quantities depend on both $\beta_{1}$ and $\beta_{2}$ so the analogous expressions are much more complex. However, the basic picture is unchanged. For all $\beta_{1}$ and $\beta_{2}$ the $f-\alpha$ spectrum obeys a simple scaling relationship with respect to $\gamma$, namely $f(\alpha)=\gamma h(\alpha / \gamma)$. In figure 1 the $f-\alpha$ spectrum is plotted for three different values of $m^{*}$.

Physically, the $f(\alpha(k))$ versus $\alpha$ curve simply suggest the existence of intertwined fractal subsets describing the measure. We find that when fragments are removed from the system at each time step there exist a range of fractal dimension $1.4641 \leq D_{f}\left(m^{*}\right) \leq 2$ and there are an infinitely manny $f-\alpha$ spectra for each $D_{f}$. All the $D_{f}\left(m^{*}\right)$ compete on equal footings to be the support on which the measure can distributed in a given realisation. This reflects that in addition to the entropy from the location of the fractal subset there is another source of entropy from $D_{f}\left(m^{*}\right)$ which is absent in the pure fragmmentation $(s=4)$ process.

As an aside, let us mention the connection between these models for $1<s<4$ and those of random sequential adsorption (rsa) (see [17] for a recent review). One can imagine that at each time step, the fragmentation event is a deposition in which $4-s$ fragments are deposited on the substrate and play no further part in the kinetics and $s$ regions of the substrate survive for future deposition. The difference between true rsa and our system is that in our system deposition can only take place in rather restricted set of position also as time proceeds the deposited particles get smaller in size. However, in the long time the pattern created looks rather like that created by the deposition of a mixture of sizes of ractangles. This is the $2-d$ variant of the $1-d$ system of mixture deposition studied by [18]. The $s=2$ version of this system studied in [19] the context of deposition of needles. In rsa, one of the interesting observables is that system reaches a jamming limit at long time which is less than random close packing. However, in this case we find instead of having a jamming limit the number density shows power law behaviour with non-trivial exponent. In general, the jamming limit can be found using the following relation

$$
\theta(t \rightarrow \infty)=1-\int_{0}^{\infty} d x \int_{0}^{\infty} d y f(x, y ; t)
$$

This gives

$$
1-\theta(t \rightarrow \infty) \sim t^{\sqrt{s}-1}
$$

To understand the role played by the dimension and shape we consider now a different model ie

$$
F\left(x_{1}, y_{1} ; x, y\right)=x^{\beta_{1}} y^{\beta_{2}} \delta\left(2 x_{1}-x\right) \delta\left(2 y_{1}-y\right)
$$


Substitute this into the rate equation to get,

$$
\frac{\partial f(x, y ; t)}{\partial t}=-\frac{1}{2^{2}} x^{\beta_{1}} y^{\beta_{2}} f(x, y ; t)+2^{\beta_{1}+\beta_{2}} s f(2 x, 2 y ; t)
$$

And the rate equation for the moments

$$
\frac{\partial M_{m, n}(t)}{\partial t}=-\left(\frac{1}{2^{2}}-\frac{s}{2^{m+n}}\right) M_{m+\beta_{1}, n+\beta_{2}}(t)
$$

This again gives infinitely many hidden conserved quantity. As before the condition for the conserved dynamical quantity can be obtained from the requirement of time independent moments

$$
m+n=2+\frac{\ln s}{\ln 2}
$$

This linear relation between $m$ 's and $n$ 's implies asymptotic power law decay of the moments with linear exponent in $m$ 's and $n$ 's in time:

$$
M_{m, n}(t) \sim A(m, n) t^{-\alpha(m+n)}
$$

Substitute this into the rate equation for the moments to give a difference equation

$$
\alpha\left(m+n+\beta_{1}+\beta_{2}\right)=\alpha(m+n)+1
$$

Iterating the above difference equation and using the appropriate boundary condition gives

$$
\alpha(m+n)=\frac{m+n-\left(2+\frac{\ln s}{\ln 2}\right)}{\left(\beta_{1}+\beta_{2}\right)}
$$

This gives us the power law decay of all the moments and allows us to show that the average number of fragments $\langle N(t)>$ and the average area are related by

$$
<N(t)>\sim<x y>^{-\frac{\ln s}{2 \ln 2}}
$$

Consequently, the fractal dimension for $s<4$ is given by

$$
D_{f}=\frac{\ln s}{\ln 2}
$$

and we have $f(\alpha)=D_{f}$ and $\tau(k)=D_{f}(1-k)$ (self-similar). In short, model B exhibits simple scaling.

These results give us the opportunity to ask why one needs an infinite number of independent exponents to characterise the scaling relations in model A while model B only exhibits scaling. To find the answer we need to go back to the nature of the models themselves and search for the things we lost in moving from the model A to the model B. In the model A we had stochastic homogenieity which implies the fragmentation of an object possesses ergodic probability distribution. In this model, two orthogonal cracks are placed independently parallel to the sides ie they can pass through any points in Euclidean space. While the model $\mathrm{B}$ describe two orthogonal cracks are allowed to place only at the middle of the objects to produce successfully four equal sized fragments. It implies that the size is 
no longer intrigued with shape ie shape is determined by the initial condition. Note that it is one of the infinitely many possibility of the former model. Thus if there is a mixture of particles of different size and shape, and if any fragments are equally likely to be picked, in the second model, once a fragments with definite shape is picked for fragmentation that will only produce of that shape. Thus it is the broken ergodicity in shape that causes the absence of multiscaling.

In conclusion, we have studied the two different models to understand the fragmentation phenomenon when there are more than one dynamical variable. We found significantly different behaviour between the two models, although both models have an infinite number of conserved moments. Interestingly, these models also helps us to explain the occurence of multifractality in fragmenting systems, which is not yet fully understood. 


\section{Acknowledgement}

MKH would like to thank the CVCP for an ORS award.

\section{References}

1. Englemen R 1991 J. Phys: condensed matter 31019

2. Mark H and Simha R 1940 Trans. Faraday Soc. 35611

3. Montroll E W and Simha R 1940 J. Chem. Phys. 8721

4. Filippov A F 1961 Theory Probab. Appl. 4275

5. Kolmogorov A N 1941 Dan USSR (Doklady) 3199

6. Ziff R M and McGrady E D 1985 J. Phys. A 183027

7. McGrady E D and Ziff R M 1987 Phys. Rev Lett 58892

8. Williams M M R 1990 Aero. Sci. and Technol. 12538

9. Cheng Z and Redner S 1990 J Phys A 231233

10. Rodgers G J and Hassan M K 1994 Physical Rev. E 503458

11. Krapivsky P L and Ben-Naim E 1994 Phys Rev E 503508

12. Boyer D Tarjus G and Viot P 1995 Phys Rev E 501043

13. Singh P and Hassan M K 1996 Phys. Rev. E in press

14. Krapivsky P L Ben-Naim E 1994 Physics Letters A 196168

15. Hassan M K and Rodgers G J Physics Letters A 20895

16. Luke Y L 1969 The special functions and their approximation 1 ( New York, Academic Press)

17. Feder J, 1988 Fractals (New York, Plenum).

18. Evans J W 1991 Rev. Mod. Phys. 651281

19. Krapivsky P L 1992 J. Stat. Phys. 69135

20. Tarjus G and Viot P 1991 Phys. Rev. Lett. 671875 


\section{Figure}

1. Three of the $f-\alpha$ spectra for model A when $s=3$ and $\beta_{1}=\beta_{2}$. The three curves are for $m^{*}=2,4$ and $\infty$. 